\title{
Orthogonal Superposition Rheometry of Colloidal Gels: Time-Shear Rate Superposition
}

Received 00th January 20xx, Accepted 00th January 20xx DOI: $10.1039 / x 0 \times x 00000 x$ www.rsc.org/softmatter

\author{
Sang Hoon Sung, $\ddagger^{\mathrm{a}}$ Sunhyung Kim, $\neq \S^{\mathrm{b}}$ Jan Hendricks, ${ }^{c}$ Christian Clasen ${ }^{* c}$ and Kyung Hyun \\ Ahn*a
}

\begin{abstract}
We explore the relaxation behavior of model colloidal gels under steady shear flow by means of orthogonal superposition rheometry. Fumed silica and carbon black dispersions in Newtonian matrices are used as a model system. As shear rate increases, the frequency dependent orthogonal moduli of the gels shift along the frequency axis without changing their shape, which finally can be superimposed to yield a single master curve. This indicates that the shear rate tunes a master clock for overall relaxation modes in the sheared colloidal gels to produce a "time-shear rate superposition (TSS)", as temperature does in polymeric liquids to produce a time-temperature superposition (TTS). The horizontal shift factor required at each shear rate to obtain the master curve is found to be directly proportional to the suspension viscosity for all the cases. From this result, we suggest that the suspension viscosity determines the overall relaxation time of the particles in the flowing colloidal gel.
\end{abstract}

\section{Introduction}

Colloidal gels composed of attractive nanoparticles are characterized by a sample-spanning network that results in a solid-like rheological behavior at rest, but yields and flows when subjected to external stresses. This yield stress and the flowability make such gels attractive for a wide range of applications, such as flowable electrodes and electrode slurries. ${ }^{1-}$ 4 Colloidal gels experience steady shear flow in various applications such as coating or painting,5,6 in which the material experiences also a variety of flow fields, resulting in microstructural changes at several different length scales. ${ }^{7}$ While it is therefore important to characterize and understand the structure and property in the steady shear flow, there have been only few studies on the 'fluidized' state of the colloidal gel using rheological methods, mainly due to limitations in the measurement techniques: viscosity and normal stress measurements provide only a macroscopic response to the steady shear flow. ${ }^{8}$ There has been a substantial amount of studies describing the microstructure of colloidal gels and its evolution under steady shear, using a variety of approaches such as simulations, ${ }^{9,10}$ scattering techniques ${ }^{11,12}$ and direct observation 10,13-15 that directly investigate the microstructure of the gel under steady shear flow. However, there has been limited information on the time-dependent viscoelastic response of the gels under steady shear, whose time scale is associated with the length scale of the microstructure of the material.

\footnotetext{
a. School of Chemical and Biological Engineering, Seoul National University, 08826 Seoul, Korea. E-mail: ahnnet@snu.ac.kr

b. Institute for Mechanical Process Engineering and Mechanics, Karlsruhe Institute of Technology, Gotthard-Franz-Straße 3, 76131 Karlsruhe, Germany

c. Department of Chemical Engineering, KU Leuven, 3001 Leuven, Belgium. Email: christian.clasen@kuleuven.be

$\dagger$ Electronic Supplementary Information (ESI) available. See DOI: 10.1039/x0xx00000x

‡ S. Sung and S. Kim contributed equally to this work.

$\S$ Current address: Corporate R\&D, LG Chem, Republic of Korea
}

Probing the viscoelastic response of colloidal gels while simultaneouslysubjecting the sample to a steady shear flow could provide necessary information to understand the link between microstructure development and macroscopic flow properties. One possibility to probe the evolution of relaxation modes under steady shear flow is the superposition of an oscillatory deformation onto a steady shear flow ${ }^{16,17}$. This method enables to analyze the mechanical response in a non-linear flow based on concepts used in linear viscoelasticity. In particular, the superposition of an oscillatory deformation in the direction orthogonal to the steady shear flow results in only a weak coupling of the two flow fields, hence the corresponding moduli can be directly related to the microstructure under flow. ${ }^{16-21}$ Utilizing orthogonal superposition rheometry (OSR), dynamics of the flow behavior in steady shear flows of various complex fluids including associative polymers, ${ }^{17}$ wormlike micelles, ${ }^{18,19}$ colloidal glasses ${ }^{20}$ and colloidal gels ${ }^{21}$ have been studied.

In this study, we present the linear viscoelastic properties of colloidal gels under steady shear flow measured simultaneously using OSR. We could identify the relaxation behavior of fumed silica gels and carbon black gels in a Newtonian matrix during 'fluidization' by an applied steady shear flow. To investigate how the relaxation timescale depends on the flow, we analyzed the frequency-dependent orthogonal viscoelastic moduli $G_{\perp}^{\prime}(\omega)$ and $G_{\perp}^{\prime \prime}(\omega)$ with respect to the increase in shear rate. A master curve was obtained by shifting the orthogonal moduli along the frequency axis in a similar manner to time-temperature superposition of polymeric liquids. ${ }^{22}$ From the shear ratedependence of the shift factor, the microscopic mechanism of the relaxation behavior of the colloidal gel under shear flow will be discussed in perspective to that of a Brownian suspension.

\section{Experimental}

\section{2-1. Materials}

In this study, we used two colloidal gels in Newtonian matrices: a fumed silica in a polybutene and paraffin oil mixture, and a carbon black in polybutenes. These are well-known model systems widely utilized to study the rheological behavior of colloidal gel 
with high reproducibility. ${ }^{21,23-25}$ The fumed silica gel was prepared by mixing hydrophobic fumed silica particles (Evonik Aerosil R972) in a mixture of $80 \%$ nonpolar oligomeric polybutene $\left(M_{n}=920 \mathrm{~g} / \mathrm{mol}\right.$, Sigma-Aldrich) and 20\% paraffin oil (Sigma-Aldrich) as the matrix. The viscosity of the mixture of polybutene and paraffin oil was determined as $0.3 \mathrm{~Pa} \cdot \mathrm{s}$ at $25{ }^{\circ} \mathrm{C}$ with Newtonian behavior over the accessible and relevant range of shear rates. The carbon black gels were composed of carbon black particles (Cabot Vulcan XC72R) dispersed in Newtonian matrices with three different viscosities. For this two polybutenes $\left(\right.$ PB320; $M_{n}=320 \mathrm{~g} / \mathrm{mol}$ and PB920; $M_{n}=920 \mathrm{~g} / \mathrm{mol}$ ) were mixed in different ratios to adjust the Newtonian viscosities for three matrices $(0.04,0.4,4 \mathrm{~Pa} \cdot \mathrm{s})$. Details on the formulation of the fumed silica gel and the carbon black gels are provided in Table 1. All the samples were dispersed with an Ultra Turrax T18 disperser (IKA, Germany) at $8000 \mathrm{rpm}$ for $5 \mathrm{~min}$. Subsequently, bubbles in the suspensions were removed using a vacuum desiccator.

Table 1 Characteristics of matrix and particles used to prepare the model colloidal gels.

\begin{tabular}{|c|c|c|c|}
\hline Particle & $\begin{array}{l}\text { Volume } \\
\text { fraction }\end{array}$ & Matrix & $\begin{array}{c}\text { Matrix viscosity } \\
\text { at } 25^{\circ} \mathrm{C}\end{array}$ \\
\hline $\begin{array}{l}\text { Fumed } \\
\text { silica }\end{array}$ & $2.5 \%$ & $\begin{array}{c}\text { PB920 and } \\
\text { paraffin oil, 8:2 by } \\
\text { weight }\end{array}$ & $0.3 \mathrm{~Pa} \cdot \mathrm{s}$ \\
\hline \multirow{3}{*}{$\begin{array}{c}\text { Carbon } \\
\text { black }\end{array}$} & \multirow{3}{*}{$3.0 \%$} & $\begin{array}{l}\text { PB920 and } \\
\text { PB320, 3:1 by } \\
\text { weight }\end{array}$ & $4 \mathrm{~Pa} \cdot \mathrm{s}$ \\
\hline & & $\begin{array}{l}\text { PB920 and } \\
\text { PB320, 1:2 by } \\
\text { weight }\end{array}$ & $0.4 \mathrm{~Pa} \cdot \mathrm{s}$ \\
\hline & & PB320 & $0.04 \mathrm{~Pa} \cdot \mathrm{s}$ \\
\hline
\end{tabular}

\section{2-2. Rheological measurements}

Orthogonal superposition rheometry measurements were performed using an ARES-G2 rheometer (TA Instrument) with a modification of the normal force control board to perform orthogonal oscillations in the shear flow field of a custom built double wall Couette geometry (shown in Fig. 1a) as described in previous works. ${ }^{18-21}$ The double wall Couette geometry features openings in the inner wall of the cup to minimize pumping flow effects. The oscillatory motion can be imposed perpendicular $\left(\dot{\gamma}_{\perp}\right.$, orthogonal superposition) to the direction of the steady or transient shear flow $(\dot{\gamma})$ as schematically shown in Fig. 1b. The oscillatory shear deformation orthogonal to the main flow is obtained by moving the Couette bob axially, whereas the main steady shear flow is achieved by rotating the Couette cup (see Fig. 1a). Detailed information on the orthogonal setup can be found in earlier work.18 Steady shear flow is applied over a shear rate range of 0.01 to $100 \mathrm{~s}^{-1}$. During steady state shear flow, smallamplitude oscillations were superimposed in the axial direction with a variable frequency. The orthogonal measurements are highly reproducible if the same experimental protocol is applied (an example or a repeat measurement is shown on Fig. $\mathrm{S} 1$ in the ESI†) (a)

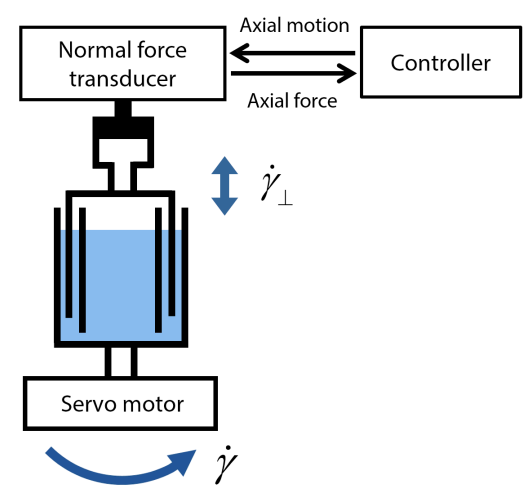

(b)

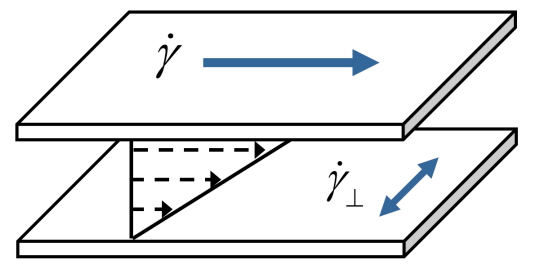

Fig. 1 (a) Schematic diagram of the orthogonal superposition setup with a double wall Couette cell. An opening is made in the inner wall of the cup that connects with a liquid reservoir in the center of the cup. (b) Superposition flows: orthogonal superposition is achieved by superposing the oscillatory flow in the vorticity direction $\left(\dot{\gamma}_{\perp}\right)$ onto the steady shear flow $(\dot{\gamma})$.

Colloidal gels do not exhibit absolute (rest) values for the moduli due to aging effects. Moreover, the linear viscoelastic moduli of the colloidal gels at rest are strongly dependent on shear history. To minimize the effect of shear history and obtain reproducible measurements, the following preconditioning protocols were applied before all measurements of frequency dependent moduli of the materials at rest: (1) preshear at $300 \mathrm{~s}^{-1}$ for $120 \mathrm{~s}$ followed by $0.1 \mathrm{~s}^{-1}$ for $300 \mathrm{~s}$, and a rest time of $7200 \mathrm{~s}$ for the fume silica gel. (2) preshear at $300 \mathrm{~s}^{-1}$ for $120 \mathrm{~s}$, followed by $0.1 \mathrm{~s}^{-1}$ for $60 \mathrm{~s}$, and a rest time of $3600 \mathrm{~s}$.

\section{Results}

\section{3-1. Fumed silica gel}

Fig. 2a shows the steady shear viscosity curve of the fumed silica gel, exhibiting a yielding behavior with a power law slope close to -1 at $\dot{\gamma}<0.1 \mathrm{~s}^{-1}$. The fumed silica gel shows a shear thinning behavior as shear rate increases, and a nearly-Newtonian plateau at $\dot{\gamma}>100 \mathrm{~s}^{-1}$. Fig. $2 \mathrm{~b}$ shows the frequency dependency of the linear viscoelastic moduli $G^{\prime}$ and $G^{\prime \prime}$, measured both in the rotational and axial direction without the application of a steady shear flow. $G^{\prime}$ is nearly independent of $\omega$ and much larger than $G^{\prime \prime}$ for the whole range of the measured $\omega$. Such an elastic behavior indicates a solid-like character of the particle network at rest. 26 With increasing $\omega$ the viscous modulus $G^{\prime \prime}$ is eventually increasing above its plateau value when approaching the high frequency regime that is controlled by the thickness of lubrication boundary layers and diffusion of the primary particle aggregates 

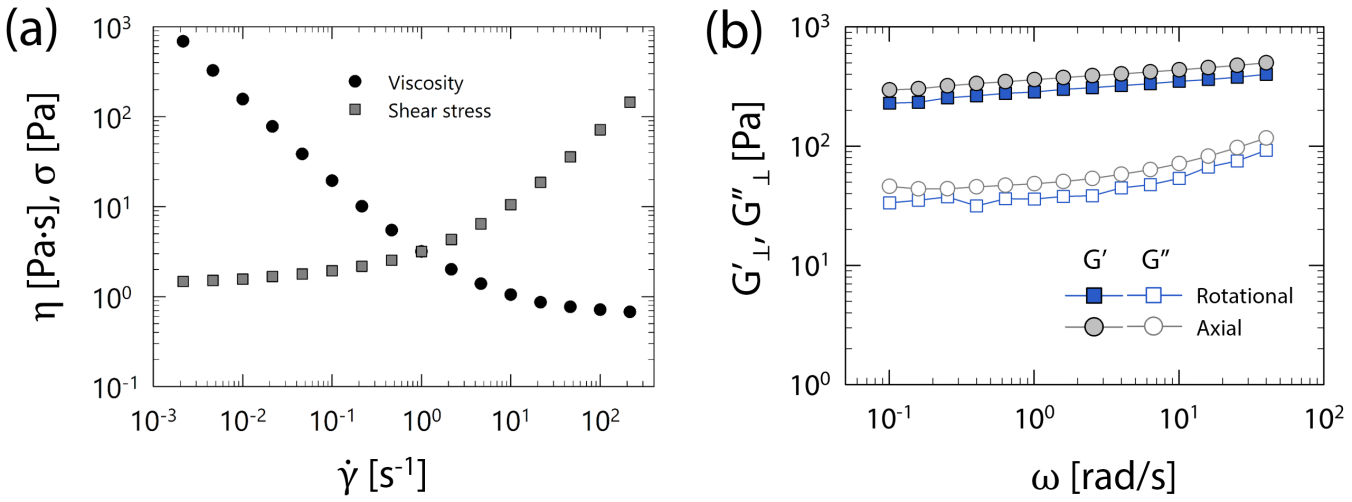

Fig. 2 (a) Steady state flow curve and (b) frequency dependent linear viscoelastic moduli at rest of a 2.5 vol\% fumed silica dispersion.

of size $a,{ }^{27}$ and thus related to the matrix viscosity $\eta_{m}$ reported in Table 1. It can be said that $G^{\prime \prime}$ is controlled by an oscillatory Peclet number $P e_{\omega}=6 \pi a^{3} \eta_{m} \omega / k_{B} T$. Colombo et al. ${ }^{21}$ recently showed that this particle network of colloidal gels exhibits a mechanical anisotropy depending on preshear conditions. The similar values of the moduli in rotational and axial direction obtained in Fig. $2 b$ indicate that this assures a sufficiently isotropic state of the material for our investigations. It should be noted that with the current experimental setup of an opened double wall Couette the measured axial moduli are always larger than the rotational moduli, which can be attributed to an increased resistance caused by pumping flows. Are recent numerical simulation of the actual flow field in this opened Couette geometry by Colombo et al.21 indicated that this error amounts for Newtonian fluids to at least $10 \%$, in agreement with our observations in Fig. 2b. This inherent difference prevents a direct comparison between axial and rotational moduli. Nevertheless, the difference due to this inherent measurement error $\left(G_{\text {axi }}^{\prime} / G_{\text {rot }}^{\prime}<2\right.$ for all the experiments in the current study) is much smaller than the difference due to the shear-induced anisotropy of the colloidal gels $\left(G_{\text {axi }}^{\prime} / G_{\text {rot }}^{\prime}>100\right.$ for high $\left.\dot{\gamma}_{\text {preshear }}\right){ }^{21}$

To understand the relaxation behavior of the fumed silica gel under the steady shear flow, we determined the orthogonal superposition moduli $G_{\perp}^{\prime}(\omega)$ and $G_{\perp}^{\prime \prime}(\omega)$ at different steady shear rates as shown in Fig 3 (a direct comparision of $G_{\perp}^{\prime}(\omega)$ and $G_{\perp}^{\prime \prime}(\omega)$ in the same plot to clearly identify the crossovers between $G_{\perp}^{\prime}(\omega)$ and $G_{\perp}^{\prime \prime}(\omega)$ is given in Fig. S 2 in the ESI $\left.\dagger\right)$. At $\dot{\gamma}<0.1 \mathrm{~s}^{-1}$,

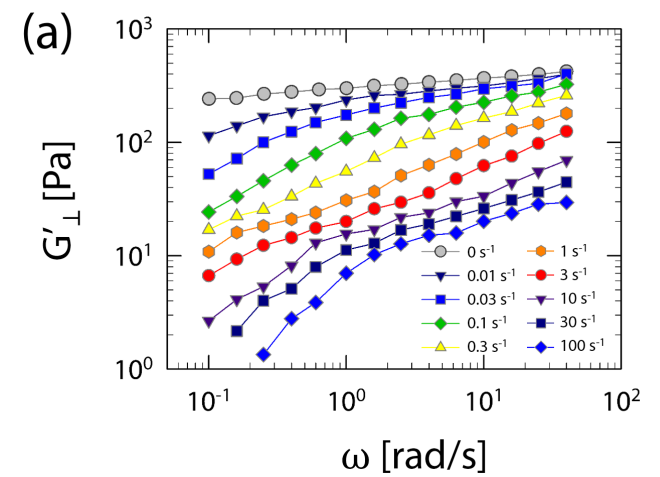

corresponding to the yielding regime in the flow curve (Fig. 2a), the curves in Fig. 3 are still similar in shape to the moduli at rest, with a plateau for both moduli, and an upturn for $G^{\prime \prime}$ when approaching the matrix viscosity $\eta_{m}$ controlled high frequency regime. However, an increase in shear rate in this regime leads eventually to a shortening of the initial plateaus and a reduction of the moduli at low frequencies as the shear rate increases, indicative of the gradual loss of connectivity in the network structure. ${ }^{28}$ At $0.1 \mathrm{~s}^{-1}<\dot{\gamma}<3 \mathrm{~s}^{-1}$, where a shear thinning behavior is observed in the flow curve in Fig. 2a, the initial plateaus in the moduli in Fig. 3 disappear and the moduli increase continuously with frequency. Further increasing the shear rate, the absolute moduli values decrease evenly over the whole frequency range. Eventually in this regime with increasing rate the decreasing moduli develop a second plateau at lower frequencies, which is roughly an order of magnitude below the initial plateau at rest. For $\dot{\gamma}>10 \mathrm{~s}^{-1}$, where a nearly-Newtonian plateau is observed in the flow curve (Fig. 2a), both $G_{\perp}^{\prime}(\omega)$ and $G_{\perp}^{\prime \prime}(\omega)$ start to deviate from the second plateau with the appearance of a terminal relaxation regime at low frequencies. It should be noted that the terminal slope $\left(G^{\prime} \propto \omega^{1.3}\right)$ is different from the ideal behavior $\left(G^{\prime} \propto \omega^{2}\right)$, because our measurements are not observing the real terminal regime due to a limitation in the frequency examined.

On closer inspection the $G_{\perp}^{\prime}(\omega)$ and $G_{\perp}^{\prime \prime}(\omega)$ curves in Fig. 3 appear similar in shape over the whole range of the shear rate, indicating that it should be possible to shift $G_{\perp}^{\prime}(\omega)$ and $G_{\perp}^{\prime \prime}(\omega)$ along the $\omega$ axis via a shift factor $a_{\dot{\gamma}}$ to obtain a single master

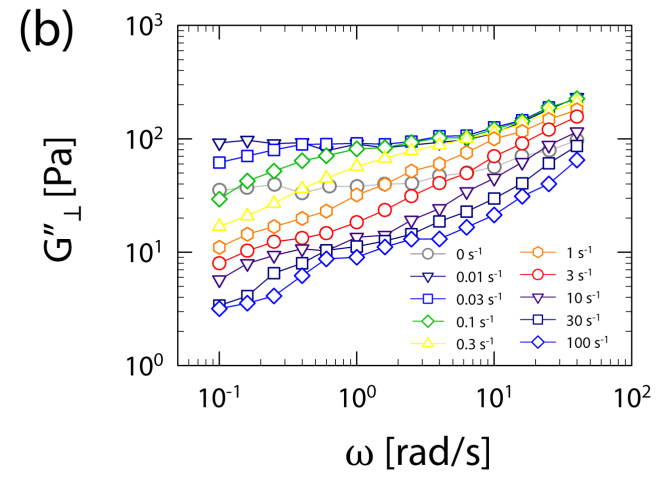



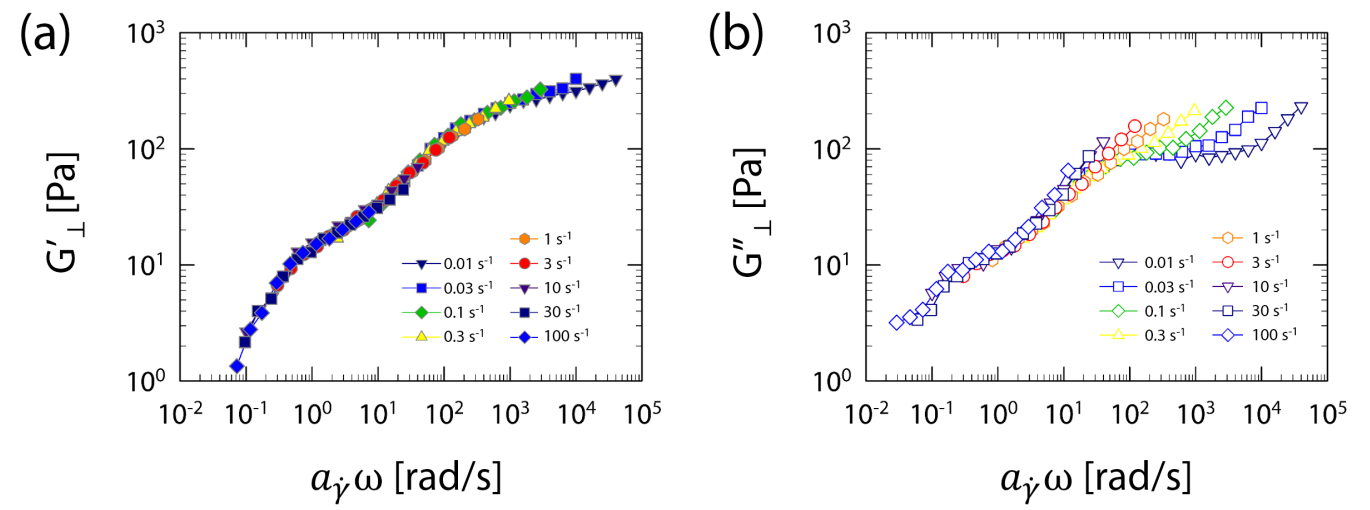

Fig. 4 Orthogonal superposition moduli $G_{\perp}^{\prime}(\mathrm{a})$ and $G_{\perp}^{\prime \prime}(\mathrm{b})$ as a function of scaled frequency $a_{\dot{\gamma}} \omega$ for different shear rates of a 2.5 vol\% fumed silica dispersion.

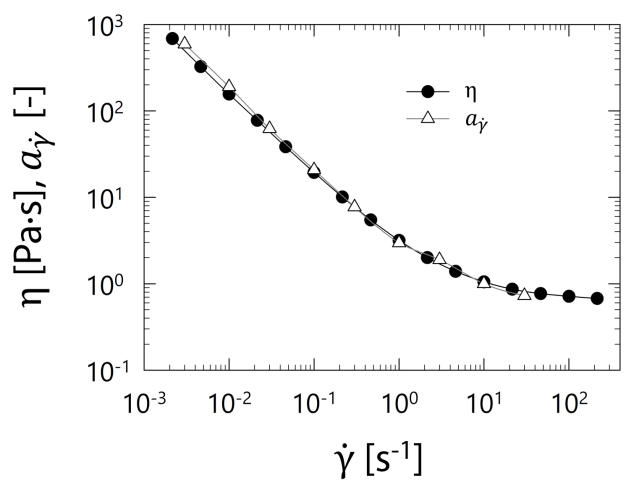

Fig. 5 Horizontal shift factor $a_{\dot{\gamma}}$ and steady shear viscosity $\eta$ as a function of shear rate of a 2.5 vol\% fumed silica dispersion.

curve (as demonstrated in Fig. 4, using the moduli at $\dot{\gamma}=10 \mathrm{~s}^{-1}$ as the reference point). The superposition of the moduli, which can be termed as 'time-shear rate superposition (TSS)', is reminiscent
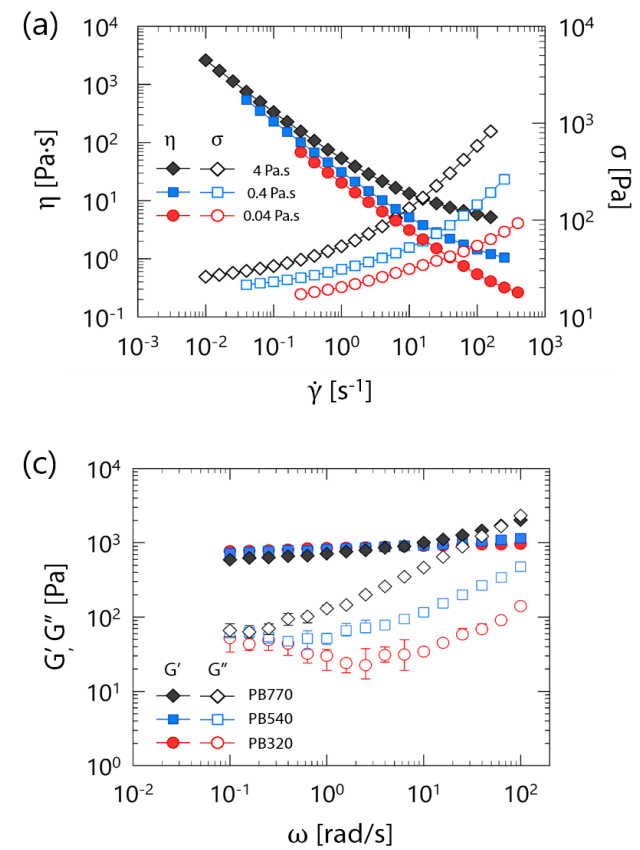

of the effect of temperature on polymeric materials where a timetemperature superposition (TTS) applies. The TSS behavior has been previously observed for a colloidal glass, where the horizontal shift factor was reported to vary linearly with the shear rate, suggesting a shear-induced relaxation behavior of the materials. ${ }^{20}$ Horizontal shifting alone was sufficient to reasonably collapse the orthogonal moduli, contrary to the case of colloidal glasses, where also a vertical shifting is necessary. A possible reason for this difference can be that the vertical shift required for the glasses reflects the effect of shear on the free volume due to the cage deformation, similar to TTS where the scaling factor represents the temperature dependence of the density. In contrast to this, there is no equivalent to a free volume in our colloidal gel systems.

In Fig. 5, the shift factor $a_{\dot{\gamma}}$ is plotted as a function of $\dot{\gamma}$ and compared to the steady shear viscosity $\eta(\dot{\gamma})$, and a remarkable similarity between the two over the entire range of investigated $\dot{\gamma}$ is observed. Furthermore, in Fig. $4 \mathrm{~b}$ we observe a deviation from the master curve for the $G_{\perp}^{\prime \prime}$ data at $a_{\dot{\gamma}} \omega>10 \mathrm{rad} \cdot \mathrm{s}^{-1}$ for shear
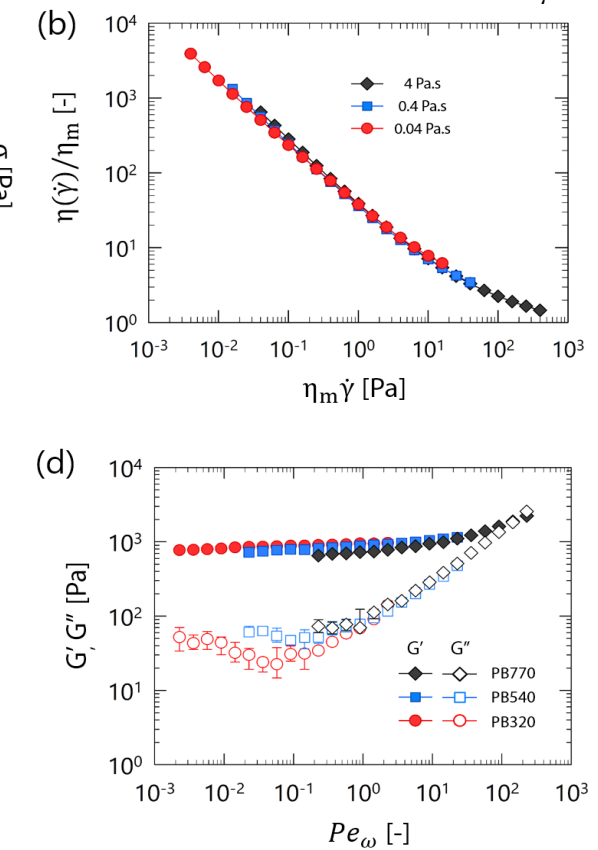

Fig. 6 (a) Steady state flow curves, (b) the flow curves normalized by the matrix viscosity (the relative viscosity as a function of the hydrodynamic stress) and (c) frequency dependent linear viscoelastic moduli (d) viscoelastic moduli versus $P e_{\omega}$ of 3.0 vol\% dispersions of carbon black in matrices with 3 different viscosities ( 0.04 , $0.4,4$ Pa s). 

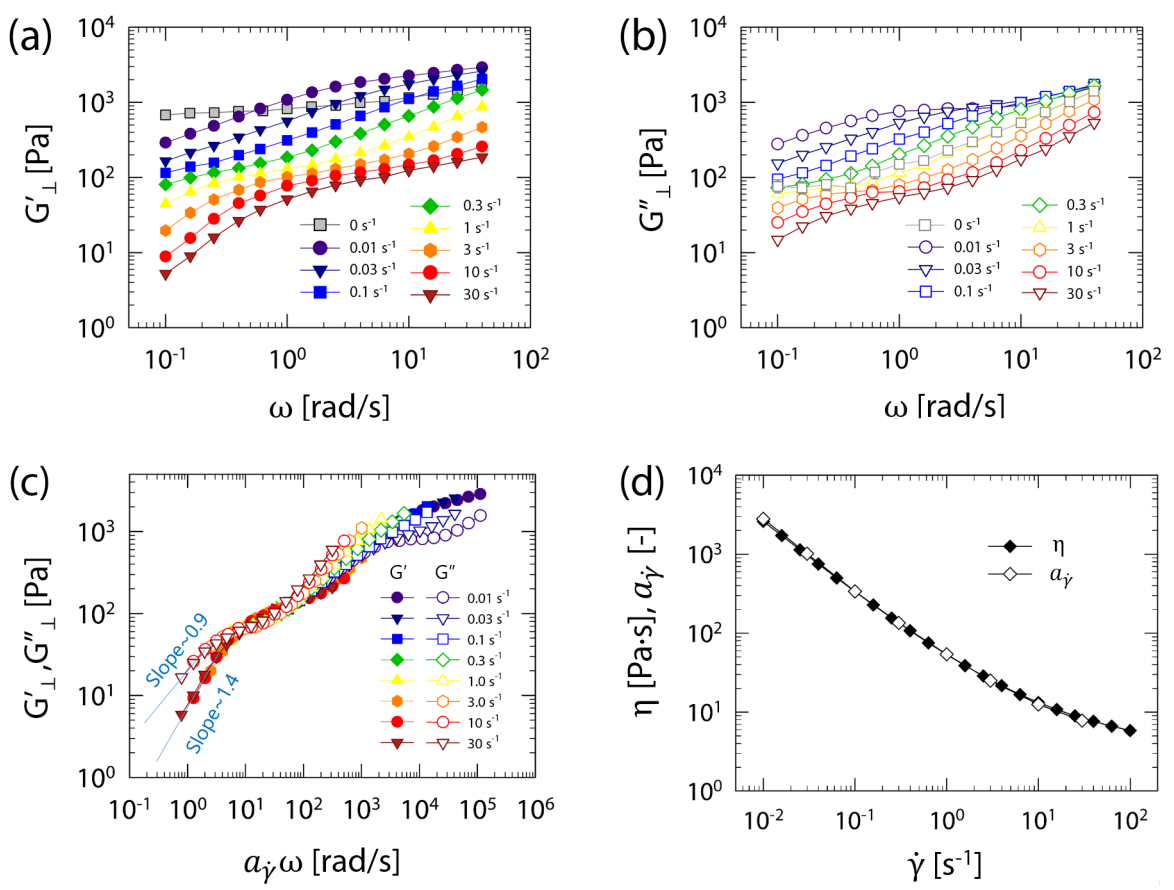

Fig. 7 Orthogonal superposition measurements at varied shear rates of 3.0 vol\% dispersions of carbon black in a matrix with $\eta_{m}=4$ Pa s: orthogonal moduli as a function of frequency $(\mathrm{a}, \mathrm{b})$ and as a function of scaled frequency $a_{\dot{\gamma}} \omega$ (c). The corresponding shift factor is shown together with the viscosity of the suspension (d).

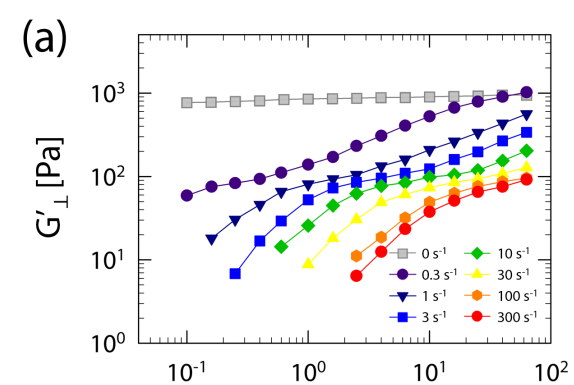

$\omega[\mathrm{rad} / \mathrm{s}]$

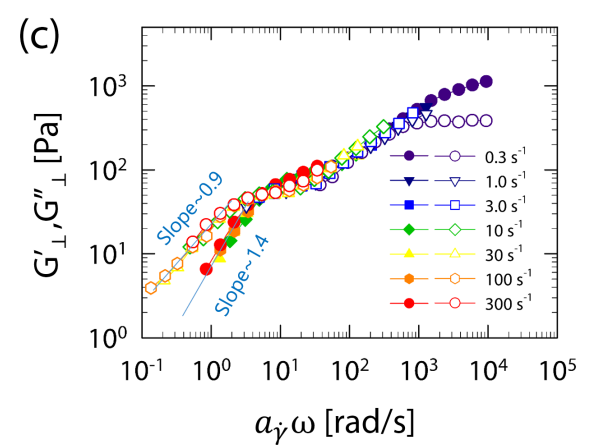

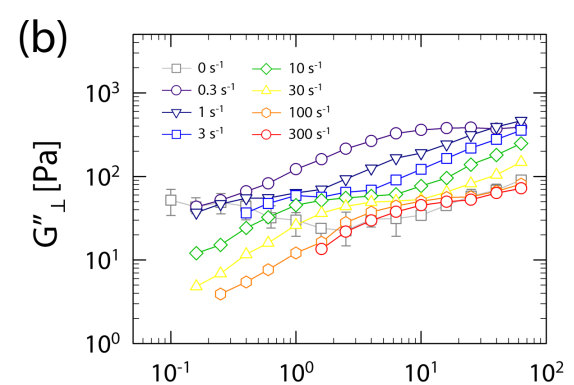

$\omega[\mathrm{rad} / \mathrm{s}]$

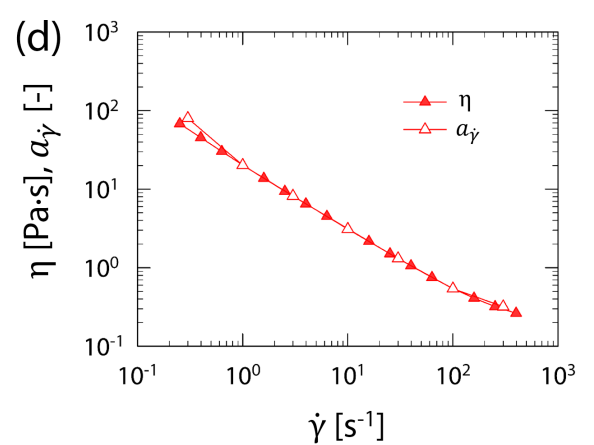

Fig. 8 Orthogonal superposition measurements at varied shear rates of 3.0 vol\% dispersion of carbon black in a matrix with $\eta_{m}=0.04$ Pa.s: orthogonal moduli as a function of frequency $(\mathrm{a}, \mathrm{b})$ and as a function of scaled frequency $a_{\dot{\gamma}} \omega(\mathrm{c})$. The corresponding shift factor is shown together with the viscosity of the suspension (d).

rates $\dot{\gamma}<10 \mathrm{~s}^{-1}$. This is not surprising since, as pointed out above, in this high frequency regime it is not the steady shear viscosity $\eta(\dot{\gamma})$, but rather the matrix viscosity $\eta_{m}$, which is represented by an oscillatory Peclet number $P e_{\omega}=6 \pi a^{3} \eta_{m} \omega / k_{B} T$ that controls the moduli increase above the initial plateau regime for the unshifted data in Fig. $3 \mathrm{~b}$ for $\dot{\gamma}<10 \mathrm{~s}^{-1}$. The nice collapse and rate independency of the $G_{\perp}^{\prime \prime}$ in this regime in Fig. $3 \mathrm{~b}$ will thus lead to a spreading of the data when scaling the frequency with an $a_{\dot{\gamma}}$ that equals the rate dependent steady shear viscosity $\eta(\dot{\gamma})$. A further investigation of this apparent viscosity control of the TSS will be shown in the next section.

\section{3-2. Carbon black gel}


To see if the TSS is a general behavior that can be observed in other material systems, and to further understand the discrepancy of $G_{\perp}^{\prime \prime}$ in the high frequency regime (Fig. $4 \mathrm{~b}$ ), we performed OSR measurement also for a different model system, carbon black gels, in which the viscosity of the matrix fluid could be systematically varied. Carbon black particles are dispersed in Newtonian matrices of three different viscosities as given in Table 1: $\eta_{m}=0.04 \mathrm{~Pa} \cdot \mathrm{s}, 0.4 \mathrm{~Pa} \cdot \mathrm{s}$ and $4 \mathrm{~Pa} \cdot \mathrm{s}$. The flow curves of carbon black gels in Fig. 6a exhibit a yielding behavior, followed by a shear thinning behavior as shear rate increases. These features are qualitatively similar with the fumed silica gel. An increase in shortening of the plateaus at low frequencies $\left(\dot{\gamma} \sim 0.01 \mathrm{~s}^{-1}\right)$, followed by a reduction over the whole frequency range $\left(0.03 \mathrm{~s}^{-1}\right.$ $<\dot{\gamma}<1 \mathrm{~s}^{-1}$ ), and again the appearance of a second plateau and then a second relaxation at low frequencies as shear rate increases $\left(\dot{\gamma}>3 \mathrm{~s}^{-1}\right)$.

Again, the orthogonal moduli curves measured at different shear rates can be shifted along the frequency axis so that they overlap onto a single curve, as shown in Fig. 7c. And also, the carbon black gel in the matrix with $\eta_{m}=4 \mathrm{~Pa} \cdot \mathrm{s}$ shows deviations from a master curve for $G_{\perp}^{\prime \prime}\left(a_{\dot{\gamma}} \omega\right)$ above the initial plateau at high frequency, similar to the fumed silica gel, whereas the $G_{\perp}^{\prime}\left(a_{\dot{\gamma}} \omega\right)$
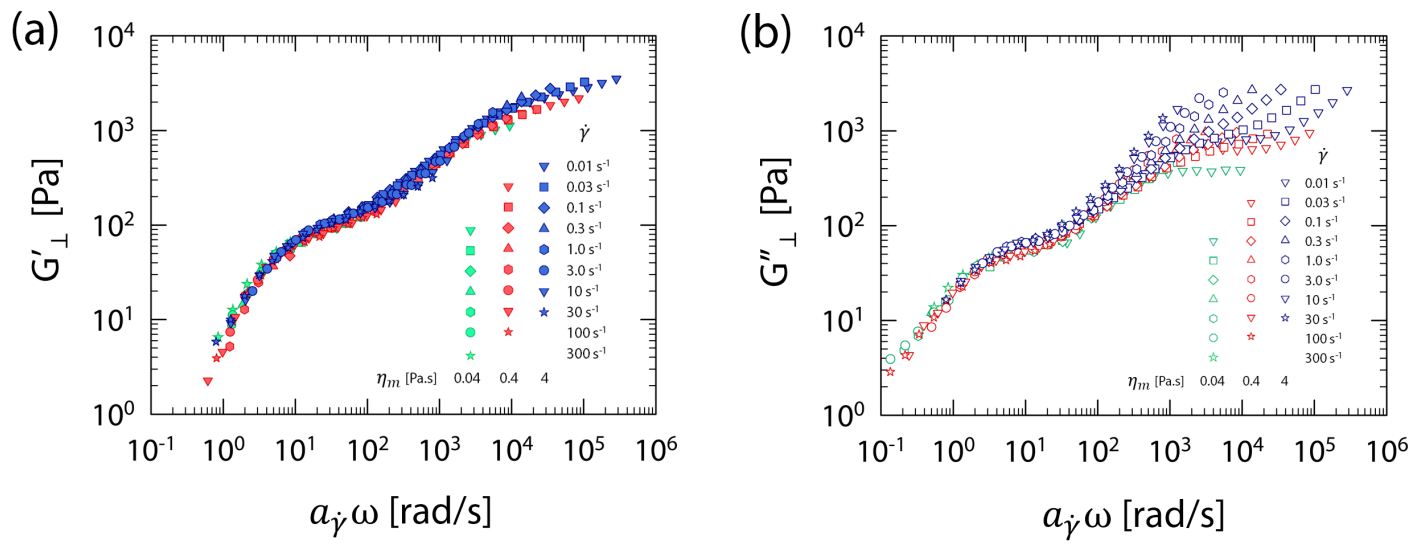

Fig. 9 Orthogonal superposition moduli $G_{\perp}^{\prime}(\mathrm{a})$ and $G_{\perp}^{\prime \prime}(\mathrm{b})$ as a function of scaled frequency $a_{\dot{\gamma}} \omega$ of 3.0 vol\% dispersions of carbon black in 3 matrices with different viscosities $(0.04,0.4,4 \mathrm{~Pa} \cdot \mathrm{s})$.

the viscosity of the matrix hardly affects the viscosity at low rates and the yield stress level. Also, viscosities coincide into nearly a single curve when they are normalized by matrix viscosities (Fig. $6 \mathrm{~b})$. The frequency dependency of the linear viscoelastic moduli $G^{\prime}$ and $G^{\prime \prime}$ is shown in Fig. 6c. Similar to the fumed silica experiments the plateau modulus $G^{\prime}$ is frequency-independent and higher than $G^{\prime \prime}$ for all three carbon black samples, indicating a solid-like behavior that is expected to be originating from the particle networks. ${ }^{29}$ It seems that the upturn of $G_{\perp}^{\prime \prime}$ is happening at lower frequencies as $\eta_{m}$ increases. This agrees with the picture that an increasing matrix viscosity increases the Brownian time of particles, as if we are looking at larger time scales. Similar to the case of viscosity, $G^{\prime}$ and $G^{\prime \prime}$ overlap when plotted against the frequency reduced by the matrix viscosity in form of the oscillatory Peclet number $P e_{\omega}=6 \pi a^{3} \eta_{m} \omega / k_{B} T$ (Fig. 6d). For the calculation of $P e_{\omega}$, we assumed a diameter of the carbon black particles or primary aggregates of $a=50 \mathrm{~nm}$ (which is obtained from the following discussion part). It should be noted that the moduli of carbon black gels at rest are particularly sensitive to the preshear. For this reason, small difference in the moduli at rest among the three samples still exists despite the well-controlled experimental protocol. In comparision to this, orthogonal superposition moduli under steady shear flow show a much lower history dependence.

In a similar way as for the fumed silica gel, OSR measurements are performed also for the carbon black gels. Fig. 7 shows the results for the $3.0 \mathrm{vol} \%$ dispersion of carbon black in the matrix with $\eta_{m}=4 \mathrm{~Pa} \cdot \mathrm{s}$. The orthogonal moduli of the carbon black gels (Fig. 7a, b) show a similar dependency on shear rate as the fumed silica gel (Fig. 3): a reduction of the moduli and data overlap perfectly over the whole frequency range. The shift factors $a_{\dot{\gamma}}(\dot{\gamma})$ used for scaling of the orthogonal moduli were again nearly identical to the steady state viscosity $\eta(\dot{\gamma})$ as seen in Fig. 7 d. Fig. 8 shows the results of the OSR measurements of the carbon black gel in the lowest-viscosity matrix $\left(\eta_{m}=0.04 \mathrm{~Pa} \cdot \mathrm{s}\right)$. The overall observations are equivalent to the case of carbon black in the highest-viscosity matrix $\left(\eta_{m}=4 \mathrm{~Pa} \cdot \mathrm{s}\right)$ (Fig. 7c), a master curve of orthogonal moduli could be obtained (Fig. 8c), by means of the steady shear viscosity $\eta(\dot{\gamma})$ as the shift factor $a_{\dot{\gamma}}(\dot{\gamma})$ (Fig. 8d). Compared to carbon black dispersed in the highestviscosity matrix $\left(\eta_{m}=4 \mathrm{~Pa} \cdot \mathrm{s}\right)$, the deviation of the $G_{\perp}^{\prime \prime}$ data from the master curve at high frequencies is not observed, which is due to the fact that the upturn of $G_{\perp}^{\prime \prime}$ above the initial plateau is happening only at even higher frequencies. To further compare the effect of the matrix viscosity to that of the steady shear viscosity, the orthogonal moduli $G_{\perp}^{\prime}$ and $G_{\perp}^{\prime \prime}$ for all carbon black gels and the three different matrix viscosities $\eta_{\mathrm{m}}$ (i.e. $0.04,0.4$ and $4 \mathrm{~Pa} \cdot \mathrm{s}$ ) are plotted together in Fig. 9 as a function of the frequency scaled with the respective steady shear viscosity $a_{\dot{\gamma}} \omega$. The elastic moduli $G_{\perp}^{\prime}$ nicely superpose onto a single curve over a wide frequency range (Fig. 9a), confirming that the matrix viscosity has little influence on the general relaxation behavior of the system. On the other hand, $G_{\perp}^{\prime \prime}$ shows a discrepancy for the high frequency data above the initial plateau in the high $a_{\dot{\gamma}} \omega$ range (Fig. 9b).

The direct comparison of the three different matrix viscosity data shows that the scaled data for $G_{\perp}^{\prime \prime}$ starts to deviate already in between the first and second plateau and is not as nicely superposing as $G_{\perp}^{\prime}$. A similar discrepancy was observed in the OSR measurement of a colloidal glass. ${ }^{20}$ In case of the colloidal glass, the origin of high frequency deviation in $G_{\perp}^{\prime \prime}$ was interpreted as 
shear-induced slowing down of in-cage dynamics. ${ }^{30}$ However, the colloidal gels do not exhibit a cage-like structure as the glass, it is thus not likely that in the current case the discrepancy at high frequencies can be attributed to a slowing down of the short time dynamics. A more likely explanation of the deviation is an instantaneous viscous response that that would simply scale with the frequency and does not shift with shear rate.

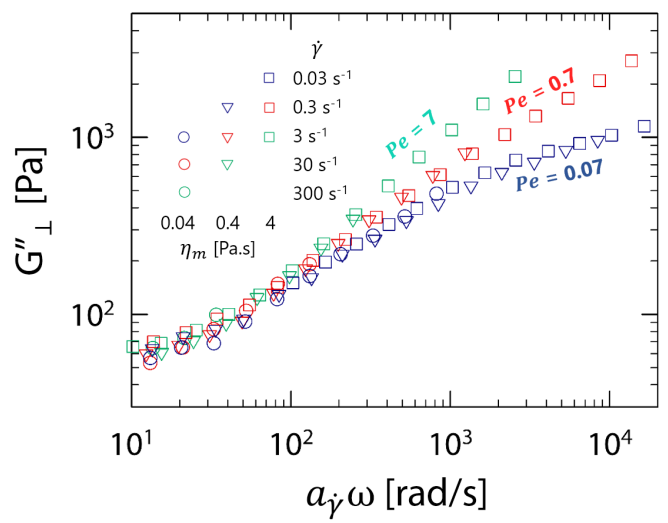

Fig. 10 Re-plotted selected data of Fig. 9b. The shear rates are selected to match three different Peclet numbers as indicated in the legend. The same color of the symbols indicates the same Peclet number.

To understand the $G_{\perp}^{\prime \prime}$ deviation regime more clearly, part of the data from Fig. $9 \mathrm{~b}$ were chosen and three shear rates for each sample were selected, such that the Peclet numbers $P e=$ $6 \pi a^{3} \eta_{m} \dot{\gamma} / k_{B} T$ of the flow are matched as $0.07,0.7$ and 7 (Fig. 10). For the calculation of the diffusivity entering $P e$, we assumed a diameter of the carbon black particles or primary aggregates of $a$ $=50 \mathrm{~nm}$ (which is obtained from the following discussion part) and the viscosity of the respective matrix fluid $\eta_{m}$. As it can be seen in Fig. 10, $G_{\perp}^{\prime \prime}$ measured for samples of different matrix viscosities but under the same Pe overlapped when plotted against $a_{\dot{\gamma}} \omega$, indicating that the discrepancy can indeed be associated to the Peclet number. Again this supports the interpretation that the deviation is due to an increasing contribution of the instantaneous viscous response on $G_{\perp}^{\prime \prime}$. The highest $P e$ samples (for example the CB 3 vol\% sample in matrix with $\eta_{m}=4 \mathrm{~Pa} \cdot \mathrm{s}$ under a rate of $30 \mathrm{~s}^{-1}$ ) exhibit indeed a high frequency slope for $G_{\perp}^{\prime \prime}$ that approaches one, similar to the slope observed for the neat Newtonian matrix.

Time-shear rate superposition of the CB dispersion is also explored with some variation of the composition (see Fig. $\mathrm{S} 3$ in the ESI†). The volume fraction of CB is varied from $1.8 \mathrm{vol} \%$ to 3.6 vol\%. All samples exhibit time shear rate superposition, and the orthogonal moduli curves are very similar in shape. These selfsimilar viscoelastic spectra over a wide range of compositions imply a self-similar microstructure of the colloidal gels ${ }^{24}$, even when subjected to shear flow.

\section{Discussion}

In this study, we found that orthogonal moduli of colloidal gels can be shifted along the frequency axis to collapse onto a single master curve, when using a shift factor in form of the steady shear viscosity of the suspension at the shear rate $\dot{\gamma}$ of the main flow for the OSR measurement. Such behavior is reminiscent of the effect of temperature on polymeric liquids, for which a timetemperature superposition (TTS) applies. This suggests that $\dot{\gamma}$ tunes a single master clock for overall relaxation modes in the system, similar as temperature does for relaxation spectra of fluids that have the same internal friction dependence of all modes on $T$. In the following, we discuss the physical mechanism of this 'time-shear rate superposition' in the colloidal gels of the present study and suggest a possible explanation why the shift factor becomes equivalent to the steady shear viscosity of the suspension.

Before discussing the microstructure of the colloidal gels subjected to different $\dot{\gamma}$, it should be noted that sheared colloidal gels display a significant mechanical anisotropy between the orthogonal and the parallel direction to the main flow, as reported recently ${ }^{21}$, caused by a spatially anisotropic breakup of the gel. Bonds in the velocity-velocity gradient plane are more easily broken, while less affected in the other planes in which the gel retains significant elasticity 21 . The frequency dependent orthogonal moduli in the present study can therefore be translated into the relaxation behavior of the gel in the velocityvorticity plane where the microstructure is less affected by the steady shear flow.

We are starting from this viewpoint of an analogy to TTS, which is described using a friction coefficient $\zeta_{0}$ that defines the relaxation time and its temperature dependence. ${ }^{34}$ Except specific cases where TTS does not suffice, ${ }^{35-37}$ the same microscopic frictional process operates at all length and time scales. ${ }^{38,39}$ In a similar fashion, a shear rate-dependent parameter that determines all relaxation times in the colloidal gel under flow can be suggested. Two hypotheses can explain the rate-dependent time scale, which is associated with the microstructure in the velocity-vorticity plane.

The first is that the shear flow changes the relaxation time of the aggregates by progressively changing the length scale of the aggregate in the velocity-vorticity plane, which has been previously suggested by Colombo et al. ${ }^{21}$ The main point of this hypothesis is that the length scale in the neutral direction of shear also breaks down gradually, though the breakdown of the aggregates mainly occurs in the velocity-velocity gradient plane. Assuming that the slow Brownian relaxation of aggregates is responsible for the viscoelastic response of the material ${ }^{31}$ and that the diffusion of the aggregates follows the Stokes-Einstein relation, rate dependent hydrodynamic radii of the aggregates $a(\dot{\gamma})$ can be calculated using the following equation (1) (for the calculated results see Fig. S 4 in the ESI $\dagger$ ).

$$
\lambda(\dot{\gamma}) \approx \frac{\pi a(\dot{\gamma})^{3}}{k_{B} T} \eta_{m}
$$

However, this hypothesis has a shortcoming: Since it assumes that the Brownian relaxation of aggregates is responsible for the viscoelastic response of the material, the change in aggregate size should affect the magnitude of the viscoelastic moduli as well as the relaxation time. As elasticity arises from the Brownian force, the change of aggregate size $r$ should result in a significant change of $G_{\perp}^{\prime}$ and $G_{\perp}^{\prime \prime}$, e.g. proportional to $r^{3}$ for Brownian hard spheres. ${ }^{32,33}$ However, the orthogonal moduli only shifts along the 
frequency axis with negligible change in their magnitude, which is inconsistent with the case where the length scale changes.

In the second approach, we hypothesize that the Brownian relaxation of the smallest unit aggregate is responsible for the orthogonal moduli. Considering that the horizontal shift factor was found to be directly proportional to the suspension viscosity at each shear rate, the viscosity can be pointed out as the parameter that determines the relaxation time of the system. Since the relaxation time of Brownian suspensions can be described by the diffusion time ${ }^{40-43} \lambda \approx \frac{\pi a l^{2}}{k_{B} T} \eta_{s u s}$, where $l$ represents a characteristic length scale, $a$ the particle radius and $\eta_{\text {sus }}$ the suspension viscosity, we can now replace the fixed suspension viscosity $\eta_{\text {sus }}$ with the rate-dependent suspension viscosity $\eta(\dot{\gamma})$, and use the particle radius as the characteristic length scale for simplicity. Here, it should be noted that $\eta(\dot{\gamma})$ is the viscosity measured in the flow direction while the orthogonal moduli are associated with the viscoelastic response in the velocity-vorticity plane that is less affected by the steady flow. As a result, the rate-dependent relaxation time $\lambda(\dot{\gamma})$ can be expressed as

$$
\lambda(\dot{\gamma}) \approx \frac{\pi a^{3}}{k_{B} T} \eta(\dot{\gamma})
$$

We calculate the relaxation time of the system from the inverse of the frequency at the minimum phase angle (or maximum $\tan (\delta)$ ) of the orthogonal measurements data (see Fig. $\mathrm{S} 5$ in the ESI $\dagger$ ). This relaxation time as a function of the suspension viscosity for the three carbon black samples is shown in Fig. 11. It can be clearly seen that the suspension viscosity and relaxation time are indeed linearly related. This explanation, that the rate-dependent suspension viscosity $\eta(\dot{\gamma})$ determines the relaxation of the colloidal gel under shear, is consistent with a previous study that showed a direct proportionality between suspension viscosity and Brownian relaxation times by theoretical 40,41 and phenomenological ${ }^{42}$ considerations. In addition, the radius of the particles can be calculated from equation (2) as $\sim 40 \mathrm{~nm}$ for the primary aggregates of the fumed silica and $\sim 50 \mathrm{~nm}$ for the carbon black, which is reasonable value previously published dimensions for these materials. 44,45

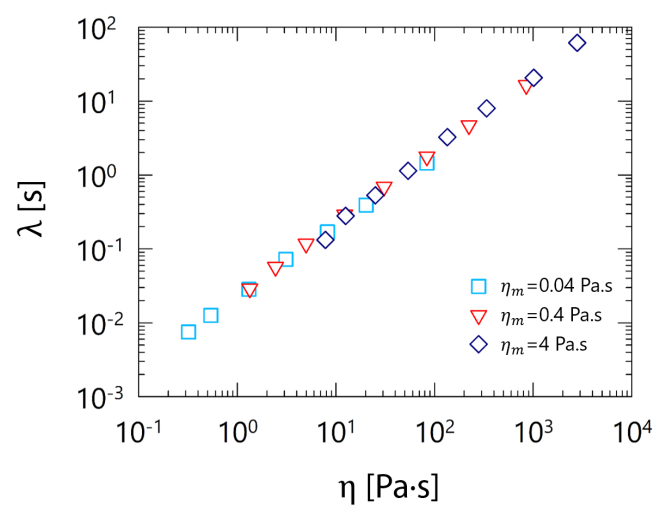

Fig. 11 Relaxation time $\lambda(\dot{\gamma})$ as a function of the suspension viscosity $\eta(\dot{\gamma})$ of the carbon black 3.0vol\% dispersions in matrices with 3 different viscosities $(0.04,0.4,4$ $\mathrm{Pa} \cdot \mathrm{s})$.

\section{Conclusion}

Utilizing orthogonal superposition rheology (OSR), we found that the orthogonal moduli of a colloidal gels under steady shear flow exhibit a time-shear rate superposition (TSS), indicating that the time scales for all the relaxation modes are equally dependent on the shear rate. From this result it can be concluded that the relaxation mechanism itself of the colloidal gel does not change with the applied shear rate, but rather the associated time scale, which is similar to a colloidal glass under flow. The horizontal shift factor for TSS is directly proportional to the rate-dependent suspension viscosity $\eta(\dot{\gamma})$. Though it will require further investigation to entirely understand the mechanism, it is suggested that $\eta(\dot{\gamma})$ determines the relaxation time of the units constituting the colloidal gel under steady shear flow. Given that the orthogonal superposition rheometry only provides mechanical information in the orthogonal direction, additional techniques such as rheo-SAXS can be utilized to assist the results. Our findings provide valuable insights for the understanding of physics controlling the flow of colloidal gels, which is difficult to directly observe otherwise. The observations also open a possibility to investigate the low-frequency behavior of weakly attractive colloidal gels that is so far limited, as aging effects usually prevent to assess the long-time scale behavior associated with the low-frequency response. While we could not measure reproducible and reliable data at very low $\dot{\gamma}$ for the materials used in the present study, it would be intriguing to examine the connection between orthogonal moduli at very low $\dot{\gamma}$ and that at rest in the future work.

\section{Conflicts of interest}

There are no conflicts of interest to declare.

\section{Acknowledgements}

C. Clasen would like to acknowledge support by the Research Foundation Flanders (FWO, project G077916N) and the People Programme (Marie Curie Actions) of the European Union's Seventh Framework Programme (FP7/2007-2013) for the support of J.H. and C.C. under REA Grant Agreement No. 607937 (SUPOLEN). K. H. Ahn acknowledges the support by the National Research Foundation of Korea (NRF) grant funded by the Korea government (MSIT) (No. NRF-2018R1A5A1024127). S. Kim acknowledges the support by NRF of Korea (No. 2013R1A6A3A03058966).

\section{References}

1 S. Jeon, H. Park, J. Yeo, S. Yang, C. H. Cho, M. H. Han and D. K. Kim, Energy Environ. Sci., 2013, 6, 1471-1475.

2 F. Y. Fan, W. H. Woodford, Z. Li, N. Baram, K. C. Smith, A. Helal, G. H. McKinley, W. C. Carter and Y.-M. Chiang, Nano Lett., 2014, 14, 2210 2218.

3 M. Youssry, L. Madec, P. Soudan, M. Cerbelaud, D. Guyomard and B. Lestriez, Phys. Chem. Chem. Phys., 2013, 15, 14476-14486.

4 G.-W. Lee, J. H. Ryu, W. Han, K. H. Ahn and S. M. Oh, Journal of Power Sources, 2010, 195, 6049-6054. 
5 S. J. Weinstein and K. J. Ruschak, Annual Review of Fluid Mechanics, 2004, 36, 29-53.

6 M. Schmitt, M. Baunach, L. Wengeler, K. Peters, P. Junges, P. Scharfer and W. Schabel, Chemical Engineering and Processing: Process Intensification, 2013, 68, 32-37.

7 J. Lee, S. Sung, Y. Kim, J. D. Park and K. H. Ahn, Current Opinion in Chemical Engineering, 2017, 16, 16-22.

8 A. Negi and C. Osuji, Rheol Acta, 2009, 48, 871-881.

9 J. D. Park, K. H. Ahn and N. J. Wagner, Journal of Rheology, 2016, 61, 117-137.

10 N. Koumakis, E. Moghimi, R. Besseling, W. C. K. Poon, J. F. Brady and G. Petekidis, Soft Matter, 2015, 11, 4640-4648.

11 A. T. J. M. Woutersen, R. P. May and C. G. de Kruif, Journal of Rheology, 1993, 37, 71-88.

12 H. Hoekstra, J. Mewis, T. Narayanan and J. Vermant, Langmuir, 2005, 21, 11017-11025.

13 K. Masschaele, J. Fransaer and J. Vermant, Soft Matter, 2011, 7, 77177726.

14 B. Rajaram and A. Mohraz, Soft Matter, 2012, 8, 7699-7707.

15 L. C. Hsiao, H. Kang, K. H. Ahn and M. J. Solomon, Soft Matter, 2014, 10, 9254-9259.

16 J. Vermant, L. Walker, P. Moldenaers and J. Mewis, Journal of NonNewtonian Fluid Mechanics, 1998, 79, 173-189.

17 J. Mewis, B. Kaffashi, J. Vermant and R. J. Butera, Macromolecules, 2001, 34, 1376-1383.

18 S. Kim, J. Mewis, C. Clasen and J. Vermant, Rheol Acta, 2013, 52, 727 740.

19 S. Khandavalli, J. Hendricks, C. Clasen and J. P. Rothstein, Journal of Rheology, 2016, 60, 1331-1346.

20 A. R. Jacob, A. S. Poulos, S. Kim, J. Vermant and G. Petekidis, Phys. Rev. Lett., 2015, 115, 218301.

21 G. Colombo, S. Kim, T. Schweizer, B. Schroyen, C. Clasen, J. Mewis and J. Vermant, Journal of Rheology, 2017, 61, 1035-1048.

22 J. D. Ferry, Viscoelastic properties of polymers, Wiley, New York, 1980.

23 K. Dullaert and J. Mewis, Rheol Acta, 2005, 45, 23-32.

24 V. Trappe and D. A. Weitz, Physical Review Letters, 2000, 85, 449452.

25 V. Grenard, T. Divoux, N. Taberlet and S. Manneville, Soft Matter, $2014,10,1555-1571$.

26 S. A. Khan and N. J. Zoeller, Journal of Rheology, 1993, 37, 12251235.

27 R. A. Lionberger and W. B. Russel, Journal of Rheology, 1994, 38, 1885-1908.

28 K. Dullaert and J. Mewis, Journal of Colloid and Interface Science, 2005, 287, 542-551.

29 Y. Aoki, A. Hatano and H. Watanabe, Rheologica Acta, 2003, 42, 209216.

30 N. Koumakis, M. Laurati, S. U. Egelhaaf, J. F. Brady and G. Petekidis, Phys. Rev. Lett., 2012, 108, 098303.

31 L. C. Hsiao, R. S. Newman, S. C. Glotzer and M. J. Solomon, PNAS, 2012, 109, 16029-16034.

32 R. A. Lionberger and W. B. Russel, Journal of Rheology, 1994, 38, 1885-1908.

33 T. Shikata and D. S. Pearson, Journal of Rheology (1978-present) 1994, 38, 601-616.

34 R. G. Larson, The Structure and Rheology of Complex Fluids, Oxford University Press, New York, 1 edition., 1998.

35 R. H. Colby, Polymer, 1989, 30, 1275-1278.

36 Y. Ding and A. P. Sokolov, Macromolecules, 2006, 39, 3322-3326.

37 S. Seiffert and J. Sprakel, Chem. Soc. Rev., 2012, 41, 909-930.

38 M. Doi and S. F. Edwards, The Theory of Polymer Dynamics, Oxford University Press, Oxford, New York, 1988.

39 A. P. Sokolov and K. S. Schweizer, Phys. Rev. Lett., 2009, 102, 248301.

40 A. J. Banchio, G. Nägele and J. Bergenholtz, The Journal of Chemical Physics, 1999, 111, 8721-8740.

41 I. S. Sohn and R. Rajagopalan, Journal of Rheology, 2004, 48, 117142.

42 R. Dannert, H. H. Winter, R. Sanctuary and J. Baller, Rheol Acta, 2017, 56, 615-622.

43 H. Watanabe, M.-L. Yao, K. Osaki, T. Shikata, H. Niwa, Y. Morishima, N. P. Balsara and H. Wang, Rheol. Acta, 1998, 37, 1-6.
44 M. J. Lázaro, L. Calvillo, V. Celorrio, J. Pardo, S. Perathoner and R. Moliner, Study and application of Vulcan XC-72 in low temperature fuel cells, 2011.

45 Z. Y. Liu, J. L. Zhang, P. T. Yu, J. X. Zhang, R. Makharia, K. L. More and E. A. Stach, J. Electrochem. Soc., 2010, 157, B906-B913. 